\title{
Multisystemic inflammatory syndrome related to COVID-19, with latent tuberculosis in bone marrow, and satisfactory response to tocilizumab, in a 7-year-old boy
}

\author{
Z. Morel', R. Suarez², E. Avalos², S. Velázquez ${ }^{3}$, F. Martínez $^{4}$, V. Lezcano ${ }^{4}$, \\ N. Astigarraga ${ }^{5}$, C. Ghezzi ${ }^{2}$, S. Brizuela 6 , G. Samudio ${ }^{4}$ \\ ${ }^{1}$ Pediatric Rheumatology, Pediatric Department, Central Hospital of the Social Welfare Institute, \\ Asunción, Paraguay; ${ }^{2}$ Pediatrics, Pediatric Department, Central Hospital of the Social Welfare Institute, \\ Asunción, Paraguay; ${ }^{3}$ Pediatric Intensive Care, Pediatric Department, Central Hospital of the Social Welfare \\ Institute, Asunción, Paraguay; ${ }^{4}$ Pediatric Infectology, Pediatric Department, Central Hospital \\ of the Social Welfare Institute, Asunción, Paraguay; ${ }^{5}$ Pediatric Cardiology, Pediatric Department, \\ Central Hospital of the Social Welfare Institute, Asunción, Paraguay; ${ }^{6}$ Pediatric Hematology, \\ Pediatric Department, Central Hospital of the Social Welfare Institute, Asunción, Paraguay
}

\section{SUMMARY}

Paediatric inflammatory multisystem syndrome temporally associated with COVID-19 (PIMS-TS) or multisystem inflammatory syndrome in children (MIS-C) is a new acute-onset systemic inflammatory disease, which mainly affects children. Latent tuberculosis infection (LTBI) is characterized by the presence of immune sensitization to Mycobacterium tuberculosis (MTB) in the absence of any clinical or radiological evidence of active disease. We present a child with MIS-C related to COVID-19, with latent TB in the bone marrow, and satisfactory response to tocilizumab. It is important to pay attention in the investigation of TB cases in countries with a high prevalence of tuberculosis, especially when opting for immunusuppression.

Key words: Multisystemic inflammatory syndrome in children; SARS-CoV-2; COVID-19; tuberculosis.

Corresponding author: Zoilo Morel

Pediatric Rheumatology

Pediatric Department, Central Hospital of the Socia Welfare Institute, Asunción, Paraguay

E-mail: zoiloma@hotmail.com

\section{INTRODUCTION}

OVID-19 has been declared a Public Health Emergency of international concern on January 30, 2020 by the World Health Organization (WHO) (1). The disease ranges from mild cough, fever, and sore throat to pneumonia, acute respiratory distress syndrome, and death (2).

Most children are asymptomatic or have mild symptoms of SARS-CoV-2 infection. However, reports from around the world support the appearance of a hyperinflammatory response in previously healthy children, related to this virus. Affected children may require pediatric intensive care and multidisciplinary evaluation by emergentologists, infectologists, intensiv- ists, cardiologists, hematologists, nephrologists, neurologists, surgeons, immunologists, and rheumatologists. This syndrome, called paediatric inflammatory multisystem syndrome temporally associated with COVID-19 (PIMS-TS) or multisystem inflammatory syndrome in children (MIS-C), shares some common characteristics with other pediatric inflammatory conditions, including Kawasaki disease, myocarditis with cardiogenic shock, syndromes of staphylococcal and streptococcal toxic shock, bacterial sepsis, and macrophage activation syndrome (MAS). Most of them occur in cases with cardiovascular shock, myocarditis, significant gastrointestinal (GI) symptoms, mild or absent respiratory symptoms, and a variable incidence of 
skin rash, red eyes, and changes in the oral mucosa, besides excessive inflammatory markers (3-5). Different studies describe cases of MIS-C related to SARS-CoV-2, mostly treated with IVIG, corticosteroids and in some cases with biologics (antiIL1R, anti-IL6R and/or anti-TNF) (6-8). Latent tuberculosis infection (LTBI) is characterized by the presence of immune sensitization to Mycobacterium tuberculosis (MBT) in the absence of any clinical or radiological evidence of active disease (9). The differential diagnosis of any severe clinical picture with multiple organ involvement should include tuberculosis (TB) (10-12).
We present the case of a patient with MISC related to COVID-19, with latent TB in bone marrow.

\section{CASE REPORT}

A 7-year-old male presented with a 10-day history of odynophagia, a 7-day history of fever, vomiting and 6-day history of diarrheal stools. He consulted on an outpatient basis on several occasions, receiving antipyretics and EV ceftriaxone. Given the persistence of the clinical picture and the worsening of the general condition, he was admitted with moderate dehydration (Tables I and II).

Table I - Laboratory tests.

\begin{tabular}{|c|c|c|c|c|c|c|c|c|}
\hline Days & $25 / 09$ & $28 / 09$ & $29 / 09$ & $30 / 09$ & $03 / 10$ & $05 / 10$ & $09 / 10$ & $13 / 10$ \\
\hline Leukocytes (4.000 - 10.000 / UL) & 3600 & 3610 & 1600 & 2000 & 1000 & 1680 & 2000 & 13,000 \\
\hline Neutrophils (\%) & 68 & 39 & 48 & 29 & 76 & 57 & 29 & 65 \\
\hline Lymphocytes (\%) & 18 & 51 & 42 & 46 & 17 & 20 & 63 & 25 \\
\hline Hemoglobin (11-16g/dl) & 8.1 & 9.5 & 10 & 8.9 & 9.3 & 7.1 & 11.1 & 11.4 \\
\hline Hematocrit (\%) & 24.1 & 27.7 & 28.6 & 25.5 & 26.1 & 19.8 & 31.7 & 32.8 \\
\hline Platelets (100.000-350.000 / UL) & 150,000 & 256,000 & 248,000 & 269,000 & 216,000 & 200,000 & 244,000 & 676,000 \\
\hline ESR $(0-20 \mathrm{~mm} / \mathrm{h})$ & & & 34 & & 15 & & 16 & 15 \\
\hline CRP (<6mg/L) & 12 & 17 & 74.5 & & 80.1 & & 18.5 & 31 \\
\hline Ferritin (21-274 ng/mL) & & & & $>15,000$ & $>15,000$ & & $>40,000$ & \\
\hline D-dimer ( $(\leq 500 \mathrm{ng} / \mathrm{mL})$ & 2364 & & & 1902 & 2536 & & 3791 & 1225 \\
\hline Ck-Mb (<25 U/I) & 5 & & & & & & & \\
\hline Troponin I (<34 ng/L) & 23.3 & & & & & & $<10$ & $<10$ \\
\hline AST (5-34 U/L) & 46 & & 43 & & & & 361 & 140 \\
\hline ALT (0-55 U/L) & 115 & & 60 & & & & 172 & 45 \\
\hline Urea (11-36 mg/dl) & 26 & & 29 & & & 35 & 21 & 49 \\
\hline Creatinine $(0,72-1,25 \mathrm{mg} / \mathrm{dl})$ & 0.40 & & 0.43 & & & 0.36 & 0.55 & 0.56 \\
\hline PT (70-100\%) / aPTT (20-40 sec) & $89 / 28$ & & $94 / 31$ & & $97 / 24$ & & $96 / 20$ & \\
\hline Coombs & $(-)$ & & & & & & & \\
\hline IL-6 ( $\leq 5.9 \mathrm{pg} / \mathrm{ml})$ & & & & & & 56.1 & & \\
\hline
\end{tabular}

ESR, erythrocyte sedimentation rate; CRP, C-reactive protein; AST, aspartate aminotransferase; ALT, alanine aminotransferase; PT, prothrombin time test; aPTT, activated partial thromboplastin time test; IL, interleukin.

Table II - Tests for SARS-CoV-2.

\begin{tabular}{|l|c|c|c|}
\hline & RT-PCR & IgM & IgG \\
\hline Patient & Negative & Negative & Positive \\
\hline Mother & Negative & Positive & Positive \\
\hline Father & Negative & Negative & Negative \\
\hline
\end{tabular}

RT-PCR, real-time polymerase chain reaction; Ig, immunoglobulin. 


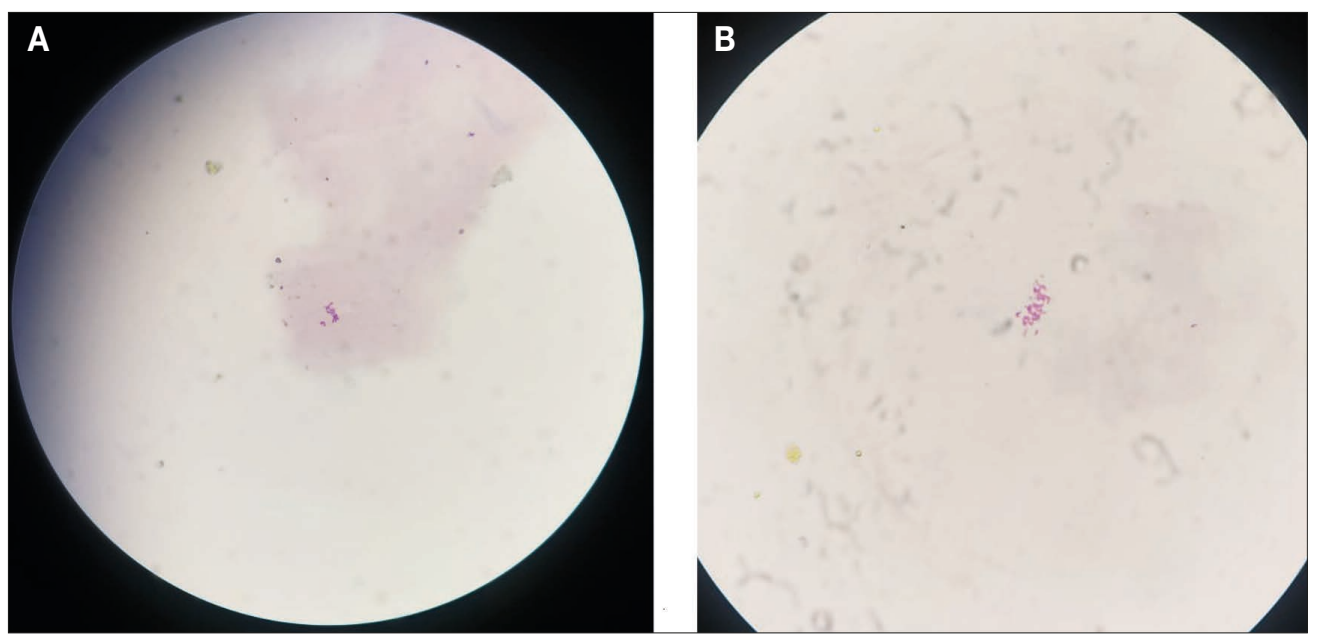

Figure 1 - A and B) Both images show acid-fast bacilli in the bone marrow.

Doppler echocardiography on the $16^{\text {th }}$ September 2020 showed slight dilation of the left ventricle with preserved systolic function, mild mitral insufficiency and moderate tricuspidal insufficiency, left coronary artery ectasia, normal pulmonary pressure, absence of pericardial effusion; no image compatible with vegetation or thrombi was observed.

The diagnosis of MIS-C related to SARSCoV-2 was made, for which he received methylprednisolone, IVIG, enoxaparin, in addition to broad-spectrum antibiotics coverage (ceftriaxone + vancomycin). After IVIG infusion at $2 \mathrm{gr} / \mathrm{kg}$, he remained afebrile for 33 hours, and again presented some fever peaks and worsening laboratory tests. Therefore, he received another dose of IVIG at $2 \mathrm{gr} / \mathrm{kg}$ again, and three boluses of methylprednisolone at $30 \mathrm{mg} / \mathrm{kg} /$ day. $\mathrm{He}$ also had high IL-6 values: $56.1 \mathrm{pg} / \mathrm{mL}$ (up to $5.9 \mathrm{pg} / \mathrm{mL}$ ), then he received tocilizum$\mathrm{ab}$, one dose at $8 \mathrm{mg} / \mathrm{kg} \mathrm{EV}$.

Bone marrow aspiration puncture was also performed, where acid-fast bacilli (AFB) were observed, without hemophagocytosis, with a decreased red series (Figure 1). Gene Xpert tests of gastric fluid were negative for M. tuberculosis. Bone marrow flow cytometry reported no increase in blasts, granulocytic series in a normal number and with complete maturation, slightly increased monocytes but without aberrant markers, CD3 + T lymphocytes in a nor- mal number and with preserved CD4:CD8 ratio.

The patient received the Bacillus CalmetteGuérin (BCG) vaccine in the first week of life.

A significant clinical improvement was observed after the administration of tocilizumab, continuing with oral prednisone, and a quadruple therapy for tuberculosis (HERZ) was started.

\section{DISCUSSION AND CONCLUSIONS}

MIS-C temporarily associated with SARS$\mathrm{CoV}-2$ is a new acute-onset systemic inflammatory disease, which mainly affects children. Definitions and diagnostic criteria have been developed in 2020 by the UK Royal College of Pediatrics and Child Health (4), the US Center for Disease Control and Prevention (CDC) (5), and the World Health Organization (WHO) (3). The criteria for the diagnosis of PIMS-TS/ MIS-C according to CDC and WHO require the demonstration of association with SARS-CoV-2 infection on the basis of history of infection, positive serological test or previous RT-PCR or antigen, or in the epidemiological context of infection in a first-degree relative with whom he lives. The required criteria are resumed in Table III, as per the WHO criteria (3).

In one of the first and largest works, Sha- 
na Godfred-Cato et al. (13) described 570 children with MIS-C related to COVID-19, grouping them into three types according to the predominant clinical findings; however, the three groups may overlap each other:

- Type 1 (35.6\%): It affects a greater number of organs and systems, especially the cardiovascular and gastrointestinal systems; it presents with abdominal pain, shock, myocarditis, lymphopenia, and marked elevation of CRP, brain natriuretic peptide (BNP or pro-BNP), ferritin, troponin. The prevalence of coronary dilations and aneurysms is higher in this group.

- Type 2 (29.6\%): This group mainly presents respiratory disorders such as cough, shortness of breath, pneumonia and ARDS, which indicates that it could be an acute and direct damage by SARS-CoV-2 or a combination with PIMS-C. This type presents the highest mortality.

- Type 3 (34.7\%): It presents mainly in the youngest patients, with skin rash and mucocutaneous lesions. The prevalence of coronary dilations and aneurysms is higher than in Type 2. This group is the one that most closely resembles Kawasaki disease, however, but with a lower prevalence of shock and myocarditis.

Our patient presented fever for 7 days, vomiting and diarrheal stools for 6 days, in addition to cardiac involvement and persistently elevated acute phase reactants, with a history of COVID-19 in the family, for which he fulfilled the criteria for MIS-C related to COVID-19 by WHO, with a mix between types 1 and 3 in his clinical presentation.

Regarding the treatment, our patient received IVIG on two occasions, in addition to methylprednisolone. However, in presence of refractory fever and elevated IL-6, he received one dose of IV tocilizumab at a $5 \mathrm{mg} / \mathrm{kg}$ dose and showed a good response. All published guidelines and studies suggest an initial use of corticosteroids and, in case of lack of response or clinical worsening, the use of biologics, especially antiIL1R (6-8, 14-15). However, in developing countries it is difficult to obtain such medi-
Table III - The WHO criteria.

\begin{tabular}{|c|c|}
\hline Age & $0-19$ years old \\
\hline Fever & Fever for $\geq 3$ days \\
\hline $\begin{array}{l}\text { Clinical } \\
\text { symptoms }\end{array}$ & $\begin{array}{l}\text { At least } 2 \text { of the following: } \\
\text { 1. rash, conjunctivitis, and mucocutaneous inflammation; } \\
\text { 2. hypotension or shock; } \\
\text { 3. cardiac involvement; } \\
\text { 4. coagulopathy; } \\
\text { 5. acute Gl symptoms }\end{array}$ \\
\hline Inflammation & $\begin{array}{l}\text { Elevated inflammation markers, including any of the following: } \\
\text { 1. } \uparrow E S R ; \\
\text { 2. } \uparrow \mathrm{CRP} ; \\
\text { 3. } \uparrow \text { procalcitonin }\end{array}$ \\
\hline $\begin{array}{l}\text { Link to SARS- } \\
\text { CoV-2 }\end{array}$ & $\begin{array}{l}\text { Evidence of COVID- } 19 \text { by the following: } \\
\text { 1. positive by PCR; } \\
\text { 2. positive by antigen test; } \\
\text { 3. positive by serology; or } \\
\text { 4. likely COVID-19 contact }\end{array}$ \\
\hline Exclusion & No obvious microbial cause \\
\hline
\end{tabular}

${ }^{*}$ Cardiac involvement is defined as the presence of myocardial dysfunction, pericarditis, valvulitis, or coronary abnormalities (including findings on echocardiogram or elevated levels of troponin/N-terminal pro-B-type natriuretic peptide). Gl, gastrointestinal; ESR, erythrocyte sedimentation rate; CRP, C-reactive protein; PCR, polymerase chain reaction.

cation, so the use of anti-IL6R or anti-TNF is more feasible. New protocols suggest classifying the patient according to their clinical presentation as mild, moderate or severe (16). This classification is based on the vasoactive-inotropic score, i.e. the degree of respiratory support, evidence of organ injury and hyperinflammation biomarkers level (17).

The WHO (18) reported 10 million new cases of TB worldwide in 2018 and 2019, however, in 2020 there has been $9,960,000$ cases with a decrease of $0.56 \%$ (19), which must be analyzed in the context of the COVID-19 pandemic. TB is a chronic persistent bacterial disease with a high burden in developing countries. The disease manifests mostly in adults with male:female ratio of 2:1 (10). WHO estimates that annually 1 million children have TB and many more harbor a latent form of infection (11). Investigation of children suspected of having TB is difficult. In clinical practice, the diagnosis requires a systematic approach that comprises 3 fundamental steps:

1) clinical history taking and detailed physical examination; 
2) imaging evaluation;

3 ) identification of the pathogen (12).

A paper published by Tadolini et al. (20) on a cohort of 49 adult patients showed that $53.0 \%$ patients had past history of TB, 28.5\% developed COVID-19 first and $18.3 \%$ patients were diagnosed with TB and COVID-19 simultaneously. The diagnosis of TB and COVID-19 was done simultaneously or within 7 days in nine patients, posing differential diagnosis challenges, suggesting that clinical assessments to investigate COVID-19 facilitated the identification of (probably pre-existing) TB.

Latent tuberculosis infection (LTBI) is characterized by the presence of immune sensitization to MTB in the absence of clinical or radiological evidence of active disease. A modeling study estimated that approximately 1.7 billion people have LTBI. Some studies showed that both mesenchymal and hematopoietic stem cells taken from individuals with LTBI or having successfully received a chemotherapeutic treatment for active TB contained intracellular MTB in a predominantly uncultivable form (21). In our patient, bone marrow aspiration was performed in the context of a study of probable hemophagocytic syndrome, with the finding of acid-fast bacilli, without other positive results, such as normal lungs, no lymphadenopathy, and even negative skin test.

BCG is a live attenuated vaccine that prevents severe tuberculosis (22). The evidence indicates that $\mathrm{BCG}$ offers protection against various non-mycobacterial and viral infections, and this might be explained in part by trained immunity (23). Miller et al. (24) published that countries with universal BCG vaccination had fewer deaths for COVID-19. In the same way, Torun et al. (25) found that BCG and MBT exposure history in healthcare workers is related to a lower mortality rate, despite the more severe disease course seen in them, which may be due to frequent exposure to tuberculosis bacillus and the mortality-reducing effects of the BCG vaccine. However, there are also two papers showing that receiving the BCG vaccine at birth does not have a protective effect against SARS-CoV-2 in- fection $(26,27)$, while the effect of a recent vaccination must be evaluated through ongoing trials.

In conclusion, we present the association of MIS-C related to COVID-19 and latent $\mathrm{TB}$, that should deserve attention in the investigation of cases in countries with a high prevalence of tuberculosis. Besides, it is important to initiate randomized controlled trials to ascertain the interactions of the various drugs used for COVID-19 and tuberculosis.

\section{REFERENCES}

1. OPS (Organización Panamericana de la Salud). La OMS declara que el nuevo brote de coronavirus es una emergencia de salud pública de importancia internacional; 30 Jan 2020. Available from: https://www.paho.org/es/ noticias/30-1-2020-oms-declara-que-nuevobrote-coronavirus-es-emergencia-salud-publica-importancia

2. Samudrala PK, Kumar P, Choudhary K, et al. Virology, pathogenesis, diagnosis and in-line treatment of COVID-19. Eur J Pharmacol. 2020; 883: 173375.

3. World Health Organization. Multisystem inflammatory syndrome in children and adolescents with COVID-19: Scientific Brief. 2020. Available from: https://www.who.int/ publications/i/item/multisystem-inflammatory-syndrome-in-children-and-adolescentswith-covid-19

4. Royal College of Paediatrics and Child Health. Guidance: Paediatric multisystem inflammatory syndrome temporally associated with COVID-19. https://www.rcpch.ac.uk/ sites/default/files/2020-05/COVID-19-Paediatric-multisystem-\%20inflammatory \%20syndrome-20200501.pdf

5. Centers for Disease Control and Prevention. Information for Healthcare Providers about Multisystem Inflammatory Syndrome in Children (MIS-C). Available from: https://www. cdc.gov/mis/hcp/index.html

6. Fernández-Sarmiento J, De Souza D, Jabornisky R, et al. Paediatric inflammatory multisystem syndrome temporally associated with COVID-19 (PIMS-TS): a narrative review and the viewpoint of the Latin American Society of Pediatric Intensive Care (SLACIP) Sepsis Committee BMJ Paediatrics Open. 2021; 5: e000894.

7. Henderson LA, Canna SW, Friedman KG, et al. American College of Rheumatology clinical guidance for multisystem inflammatory syndrome in children associated with SARSCoV-2 and hyperinflammation in pediatric 
COVID-19: Version 2. Arthritis Rheumatol. 2021; 73: e13-e29.

8. McArdle AJ, Vito O, Patel H, et al. BATS Consortium. Treatment of multisystem inflammatory syndrome in children. N Engl J Med. 2021 [Epub ahead of print].

9. Mayito J, Andia I, Belay M, et al. Anatomic and cellular niches for mycobacterium tuberculosis in latent tuberculosis infection. J Infect Dis. 2019; 219: 685-94.

10. Bandyopadhyay A, Palepu S, Bandyopadhyay K, Handu S. COVID-19 and tuberculosis coinfection: a neglected paradigm. Monaldi Arch Chest Dis. 2020; 90 (3).

11. Thomas TA. Tuberculosis in children. Pediatr Clin North Am. 2017; 64: 893-909.

12. Carvalho I, Goletti D, Manga S, et al. Managing latent tuberculosis infection and tuberculosis in children. Pulmonology. 2018; 24: 106-14.

13. Godfred-Cato S, Bryant B, Leung J, et al. California MIS-C Response Team. COVID19-associated multisystem inflammatory syndrome in children - United States, March-July 2020. MMWR Morb Mortal Wkly Rep. 2020; 69: 1074-80.

14. Cattalini M, Taddio A, Bracaglia C, et al. Childhood multisystem inflammatory syndrome associated with COVID-19 (MIS-C): a diagnostic and treatment guidance from the Rheumatology Study Group of the Italian Society of Pediatrics. Ital J Pediatr. 2021; 47: 24.

15. Hoste L, Van Paemel R, Haerynck F. Multisystem inflammatory syndrome in children related to COVID-19: a systematic review. Eur J Pediatr. 2021; 18: 1-16.

16. Jonat B, Gorelik M, Boneparth A, et al. Multisystem inflammatory syndrome in children associated with coronavirus disease 2019 in a children's hospital in New York City: patient characteristics and an institutional protocol for evaluation, management, and follow-up. Pediatr Crit Care Med. 2021; 22: e178-91.

17. McIntosh AM, Tong S, Deakyne SJ, et al. Validation of the vasoactive-inotropic score in pediatric sepsis. Pediatr Crit Care Med. 2017; 18: $750-7$.
18. World Health Organization. Global tuberculosis report; 2019. Available from: https://apps.who.int/iris/bitstream/hand le/10665/329368/9789241565714-eng.pdf

19. World Health Organization. Global tuberculosis report; 2020. Available from: https://apps. who.int/iris/bitstream/handle/10665/336069/9 789240013131-eng.pdf

20. Tadolini M, Codecasa LR, García-García JM, et al. Active tuberculosis, sequelae and COVID-19 co-infection: first cohort of 49 cases. Eur Resp J. 2020; 2001398.

21. Mayito J, Andia I, Belay M, et al. Anatomic and Cellular Niches for Mycobacterium tuberculosis in latent tuberculosis infection. J Infect Dis. 2019; 219: 685-94.

22. Batu ED, Özen S. Implications of COVID-19 in pediatric rheumatology. Rheumatol Int. 2020; 40: 1193-213.

23. Lobo N, Brooks NA, Zlotta AR, et al. 100 years of Bacillus Calmette-Guérin immunotherapy: from cattle to COVID-19. Nat Rev Urol. 2021; 18: 611-22.

24. Miller A, Reandelar MJ, Fasciglione K, et al. Correlation between universal BCG vaccination policy and reduced morbidity and mortality for COVID-19: an epidemiological study. medRxiv. 2020. [Epub ahead of print].

25. Torun S, Ozkaya S, Şen N, et al. The Relationship between COVID-19 severity and Bacillus Calmette-Guérin (BCG)/Mycobacterium tuberculosis exposure history in healthcare workers: a multi-center study. Pathog Glob Health. 2021: 1-7. [Epub ahead of print].

26. Chaisemartin C, de Chaisemartin L. Bacille Calmette-Guérin vaccination in infancy does not protect against coronavirus disease 2019 (COVID-19): evidence from a natural experiment in Sweden. Clin Infect Dis. 2021; 72: e501-5.

27. Patella V, Delfino G, Bruzzese D, et al. The bacillus Calmette-Guérin vaccination allows the innate immune system to provide protection from severe COVID-19 infection. Proc Natl Acad Sci USA. 2020; 117: 25205-6. 